\title{
Traffic safety as a factor of improving logistics in construction sector
}

\author{
Slobodan Ognjenovic ${ }^{1, *}$, Tatiana Sazonova ${ }^{2}$, Riste Ristov ${ }^{3}$, Ivana Nedevska ${ }^{4}$ and Anton \\ Kibkalo $^{5}$ \\ ${ }^{1}$ University Ss. Cyril and Methodius in Skopje, 1000, Partizanski odredi, 24, Skopje, Republic of \\ Macedonia \\ ${ }^{2}$ St. Petersburg State University of Architecture and Civil Engineering, 2-Krasnoarmejskaja, 4, Saint- \\ Petersburg, 190005, Russia \\ ${ }^{3}$ Prostor DOO-Kumanovo, 1300 Mosa Pijade 2, Kumanovo, Republic of Macedonia \\ ${ }^{4}$ Geing Krebs und Kiefer-Skopje, 1000, Boris Trajkovski str., Skopje, Republic of Macedonia \\ ${ }^{5}$ Peter the Great St. Petersburg Polytechnic University, 195251 Polytechnicheskaya str. 29, St. \\ Petersburg, Russia
}

\begin{abstract}
Security is a complex problem. It includes the driver's behavior, the features of the vehicle, those of the road and the driving conditions. Security can be approached from the aspect of the vehicle and pavement design, of driving habits, traffic regulation and application of laws. The good coordination of the driver, the vehicle and the road enables for better estimation of the traffic safety relations and interrelations giving the possibilities of improvement of the situation at present and in future. The driver has the main role in the determination of the success or failure of the road system. Over $90 \%$ of the accidents are due to improper behavior of people in traffic. Understanding of the human factors is the key factor in the optimal road design and traffic flow. Nevertheless, engineers need more understanding about driving behavior, as they are responsible for road design, for the traffic flow, and for the regulations intended to avoid errors wherever possible. The purpose of this paper is to inform about the current situation both in practice and in the researches of traffic safety, as well as to yield a broader review of the actual traffic safety situation on the roads in Republic of Macedonia.
\end{abstract}

\section{Introduction}

The terms of active and passive safety have been used in the field of transport since the 60ties. Passive security can be improved much easier and for much lower price than active safety. Therefore, more attention is paid to improvement of passive safety.

Actice safety is becoming ever more essential in improvement of traffic safety. It includes the following:

- Dynamic sefety

- The behaviour of the driver

* Corresponding author: ognjenovic@gf.ukim.edu.mk 
- Perception/visibility

- Ergonomy

- Local conditions in the vehicle (air conditioner etc.),

whereby the driver's behaviour is the key source in the improvement of active safety in view of prevention of traffic accidents [1-6].

The improper behaviour of the driver is the main reason of accidents besides the environment influences. It results from the lack of interaction betweent he driver and the vehicle and/or failure to understand the situations regarding the relation between the road and ghe environment [7-10].

In this reference, the most efficient methods of road safety improvement is the better interaction betweent he driver and the vehicle and designing roads on which the features relevant for driving are visible, understandable and well-designed, based on driving dynamism, and on the driver's behaviour from the psychological point of view [11-14].

\section{Levels of action in driving}

This basic relation can be seen on Figure 1 .

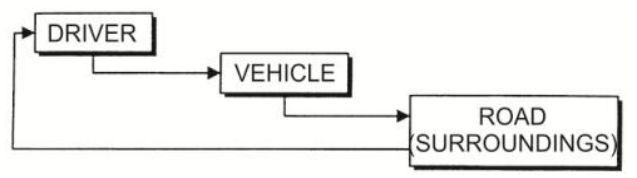

Fig. 1. Schematic presentation of the interaction driver - vehicle - road (surroundings).

Driving is mainly influenced by the applied and used information. Perceived information is compared to the information processed by the driver, which is followed by bringing a decision and taking action. Driving includes many discrete and related activities consisting of three levels of action:

-Control

-Guidance

-Navigation

Control: Control includes all activities related to interaction between the driver and the environment, as steering and velocity control, and the performance of the actions starting from the relatively simple (as for example, on the passenger vehicles) to more complex ones (as in the vehicles of several gears and clutches). Information is provided from the sense about the vehicle, from tis displays and from the pavement. Drivers perform accurate adjustments and and controls according tot he vehicle reactions. Once mastered, most control activities are performed automatically with little conscious effort.

Guidance: On this level the major activities of the driver are the maintenance of safe velocity and appropriate trajectory related toth e road and the traffic elements, as a response to the road and traffic conditions. Guidance activities are characterised by reasoning, estimation and foreseeing, in a dynemic and constrantly changing environment.

Navigation: The navigation level consists of a pre-journey phase, the one of planning the journey and choosing the route, and a journey phase that icludes the following of the previously made plan $[14,15]$.

All three levels are graphically presented on figure 2 


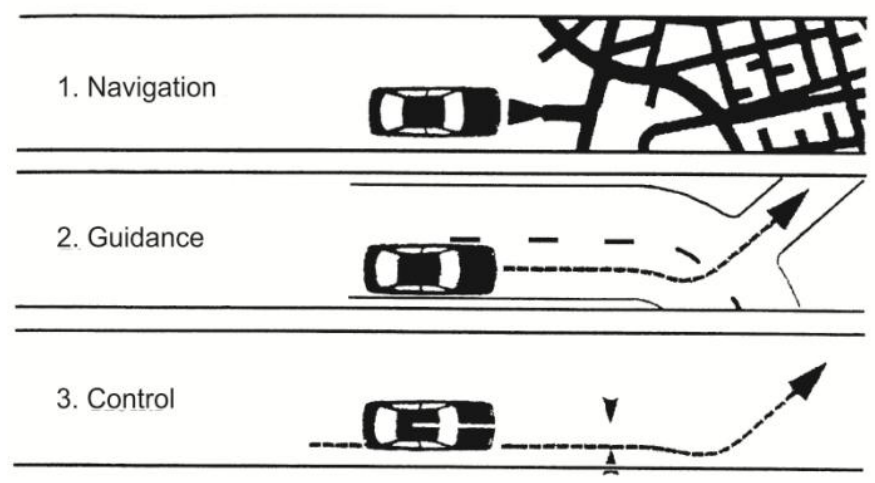

Fig. 2. Levels of action in driving.

\section{Gathering of information}

On eof the mosty important driving skills is th eperception of the lateral (side) force created upon change of the driving direnction. The level to which the kinesthetic perception is relation to visual perception has no been clearly established. The capacity of save driving with high speeds can be largely influenced by the itneraction of these two senses. Drivers usually choose the velocity before taking a turning. If the curve is an unusual one, this choice can rely on visual perception. Therefore, when the driver selects the velocity, they envisage the lateral force that is to be felt in the curve and, refering to it, the velocity os going to be reversely proportional. The research result indicate a strong reverse relation between velocity and lateral acceleration for the velocities of over $32 \mathrm{~km} / \mathrm{h}$. The lateral force created in the curb is the first criterion in the chouce of velocity. The kinesthetic perception of the lateral force is the first option by which the driver receives information that will be used in driving. There is itneraction between this kinesthetic perception and the visual perception developped fast while the driving skill is being learnt, and can be degraded by reasons such as drugs, alcohol and ageing. [16]

The velocity that is too high for a certain situaiton is the main reason of accidents causing considerable material damage and injuries. Generally speaking it can not be supposed that the driver is consciously rushich towards danger if they choose high velocity. It could be said that the driver, as pursuant to their subjective assessment of the situation, estimates the danger and selects the velocity that they consider as appropriate. Ideally, the driver would be able to control the vehicle velocity by a speedometer, compare that velocity with the necessary one and coordinate both velocities.

In reality, however, the the value of the desired velocity, as based on the estimation of the driver's risk, is suspicious. For example, the necessary velocities depend on subjective factors, as the psychological state, the need to arrive sooner, drug abuse, etc. On the other hand, the actual velocity is controlled to esentially lower level by a speedometer. This means that the driver feels only by its senses, sight being the most important of them, while the role of the kinesthetic (movement) and the haptic (touch) is a supplemental, not a minor one.

\section{Reaction time}

The reaction time has an important role in the driver's mental capacity. Tghis is the time lapse from the receipt of information, its processing until the action taken. Reaction time 
varies from one driver to another, and is the function of readiness, complexity and expectance.

Complex decisions take longer reaction time compared to the simple ones. Reaction time and the driver's behaviour have similar effect. The longer the reaction time, the greater the possibility of an error while driving [17].

The relation between the reaction time in seconds and the copmplexity, expressed in bits, is given on figure 3 on an average $85 \%$ driver. A bit is the information necessary to solve the demur between two equal probabler eactions. The picture demonstrates that even the 0-bit decision (one alternative) should be developed in a certain time and that the reaction time increases exponentially. Only a few drivers can process 3-bit or 4-bit information (8 or 16 alternatives), as a transitory decision [18].

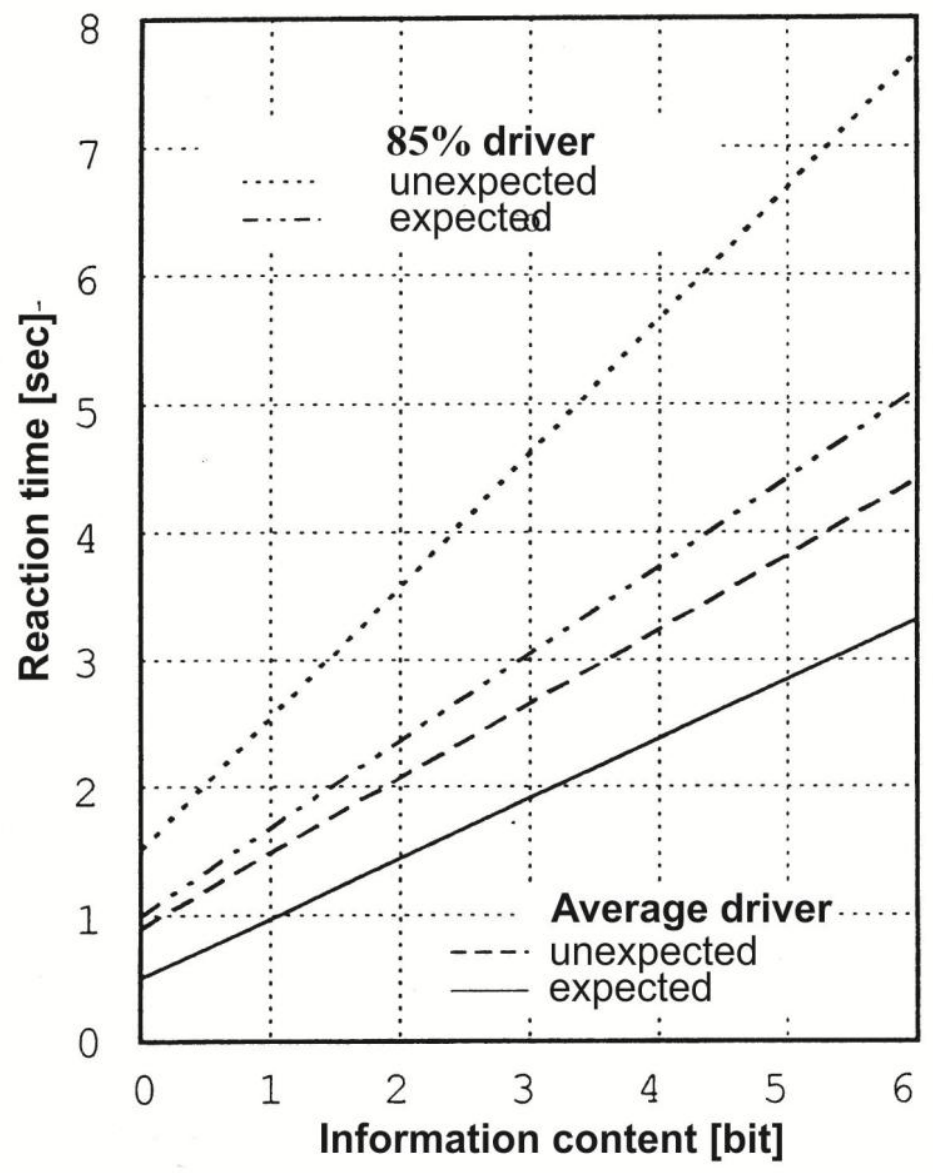

Fig.3. The reaction time of response between average and $85 \%$ of drivers.

Besides, the processing of a complex decision requires time and attention, apart from the other necessary sources of information. Thus, in situations requiring more detailed proecssing, it is usually more necessary or faster to bring several simple decisions, than one complex one. This means that the road design should be as simple as possible, without any complex sequences of elements and without many intersections. All important interactions referring tot he reaction time and the obvious distance should be processed in detail from the design point of view [19]. 


\section{Traffic accidents coused by the driver's error}

Many researches have been conducted in traffic psychology, their primary purpose being the establioshent of the psycological factors contributing to human error in driving. The principal feature of the locations with a large number of accidents is the fact that important or unusual requests are placed considering those locations regarding the processing of information on the drivers. The inefficient operaitons and the eccidents usually occur where yit is difficult to cope with the information. On locations where the road design is worse, the possibility of error and improper behaviour of the driver increases.

\subsection{Error due to information ambiguity or to confusing information}

The highest number of drivers' errors is due to false interpretation of the traffic situations and inattention. Such situations result from bad design solutions that can confuse the driver in the moment of decision-making. This phenomenon is especially critical on curves and in transitional curves and is called illusive curve.

That is a curve that does not look so sharp a sit is and the need to reduce speed can be completely disregarded. This is manifested by hard braking in the curve or, in extreme cases, by accidents. Thus, there is a divergence between the physical parameters of the curve and the psychological and physical reaction to the curve in the drivers. On subjective level, the driver understands that they enter the curve too fast. Therefore, it is supposed that an illusive curve creates a discrepancy between the physical parameters of the curve and its psycho-physical correlates. In such case, accidents result from the divergence between the physical and perceived curve, the lattrr influencing the velocity on entering the said curve.

Thus, regardless of the real curve, the partly conceald one looks "shallower" than the more visible curve with the same radius. It can be concluded that a curve with a large number of accidents is the less visible one, and not the curve similar to th eone with lower number of accidents.

This raises the question about the determinants of the driver's decision to reduce speed before a curve and the perceptive factors related to accidents. In reference therewith, the perceived curve sharpness seems to be the main determinant causing the drivers to reduce speed. The perceived width comes immediately after. Therefore, drivers should reduce speed in front of curves that look sharper, narrower and closer. The location of the driver related to the curve shuld also be taken into account. Obviouslym the driver does not possess all information necessary to estimate the real length and angle of the curve.

The following picture shows the edges and the central lines in a curve with a low number of accidents and in one where the number of accidents is high. The sharper the curve, the smaller the angle from the internal perspective.

The angle of the outer and of the inner perspective decreases with the increase of the real cruve angle (that is, the curve sharpening). However, for a given horisontal curve, the perspective angle is also under the influene of the vertical inclination and the level of elevation. Generally, the vertical curbe with a flexion increases the perspective angle, and reduces the hip-shaped curve making it look sharper.

\subsection{Error due to site requirements}

The improper motivation of the driver seems to be the main reason for accident. But, the potential road dangers, especially upon high speeds, indicate the necessity of care and readiness. If we do not suppose that people are very unreasonable, the failure of the motivational attitudes regarding the reduction of accidents indicates that the reasons for accidents can be such that even highly motivated people often fail to avoid errors. The 
basic conclusion reveals that there must be other cognitive mechanisms so strong, that they are able to surpass motivation as the main cause for errors.

\subsection{Error due to reduced driver's capacity}

Many erors are caused by inadequate capacities of of the drivers faced with an inadequate design or difficult situations. They contribute to accidents as their "secondary factors". For example, insufficient training often leads to incapacity to cope with the situation leading to slipping of the vehicle. Similarly, inadequate perceptory behaviour and taking risks can also lead to a driver's error.

Physical disabilities have limited influence on errors in driving. This is explained by the fact that the physical ability tests, for example the sight test within the procedure of acquiring a driver's license, eliminates an important part of possible accidents due to physical disabilities. On the other hand, drivers with such disabilities, as for example, elderly ones, can compensate them by driving carefully.

\subsection{Characteristics of drivers by age groups}

The highest accident risk is the one with the 18-24 years of age group of drivers. Insufficient experience, and the willingness of younger drivers to take higher risk are the main reasons thereof.

On the other hand, the number of accidents is lower in "more elderly" drivers (aged over 65 years). Their motorical capacities are more or less reduced, their eyesight, hearing capacity and memory are lower. They compensate these disadvantages by drivign slowlier, by avoiding stressful situations and relying on excperience. But the number of accidents is considerably high with this group of drivers as well, and they mostly include chain crashes and left-side turnings on intersections.

\section{Conclusions}

The purpose of this paper was to indicate the general mechanisms, from the theoretical point of view, regarding the adjustments of the drivers' behaviour related to sefety measures. A reasonable theory on the process of adaptation could, in special circumstances, be the basis of a homeostatic model of risk. The theory should not be defined as pursuant tot he accident. On the contrary it should foresee the accident level. From the theoretical point of view, it is possible to bring the following conclusions:

1. The mechanisms of adaptaiton are phenomena that are noticeable and have positive influence on security

2. The adapttion mechanisms can be observed as related tot he hierarchical stages of driving and a process to obtain results as a function of time should be developped

3. The notion of risk lies in the center of the theories explaining adaptation processing, and this notion has its objective and subjective component,

4. The subjective risk lies in the relation between the motivational components of the driver and the perception of the situaiton in which the driver is placed at a certain time.

5. The adaptation mechanisms are assisted by:

- The overestimation of the driver's own capacities, as in the perception function,

- The decision to accept high lisks,

- The incapacity of appropriate risk estimation. 


\section{References}

1. R. Lamm, B. Psarianos, T. Mailaender, Highway Design and Traffic Safety Engineering Handbook (McGraw-Hill, New York, 1999)

2. N. Moray, Attention and Mental Models, Ergonomics, 33, 365-372 (1990)

3. A. S. Cohen, Journal for Traffic Safety, 2, 125-129 (1997)

4. National Bureau of Statistics of the Republic of Macedonia (Transport and other Communications, Skopje, 2013)

5. Z. Zafirovski, N. Vatin, Applied Mechanics and Materials, 725-726, 349-354 (2015)

6. Z. Zafirovski, N.Vatin, Applied Mechanics and Materials, 725-726, 214-219 (2015)

7. M. Jocovic, B. Melovic, N. Vatin, V. Murgul, Applied Mechanics and Materials, 678, 644-647 (2014)

8. V.V Okrepilov, M.V. Leonidovich, Asian Social Science, 11 (7), 312-325 (2015)

9. V.V Okrepilov, V.L. Makarov, A.R. Bakhtizin, S.N Kuzmina, Economy of Region, 2, 301-313 (2015)

10. V.L. Kvint, V.V Okrepilov, Herald of the Russian Academy of Sciences, 84 (3), 188200 (2014)

11. V.V Okrepilov, V.N. Krutikov, G.I. El'kin, Measurement Techniques, 57 (2),109-116 (2014)

12. B. Kovačič, R. Kamnik, A. Štrukelj, N. Vatin, Procedia Engineering, 117 (1), 800-806 (2015)

13. B. Kovačič, R. Kamnik, M. Premrov, Survey Review, 43 (320), 150-161 (2011)

14. B. Kovačič, R. Kamnik, A. Štrukelj, Promet - Traffic - Traffico, 22 (1), 15-22 (2010)

15. V.V Okrepilov, A.Yu. Smirnov, Measurement Techniques, 56(1), 54-60 (2013)

16. V.V Okrepilov, Studies on Russian Economic Development, 24(1), 35-42 (2013)

17. V.V Okrepilov, Standarty i Kachestvo, 10, 52-55 (2005)

18. V.V Okrepilov, Ivanova, G. Standarty i Kachestvo, 12, 62-68. (2004)

19. V.V Okrepilov, Standarty i Kachestvo, 3, 94-96. (2003) 